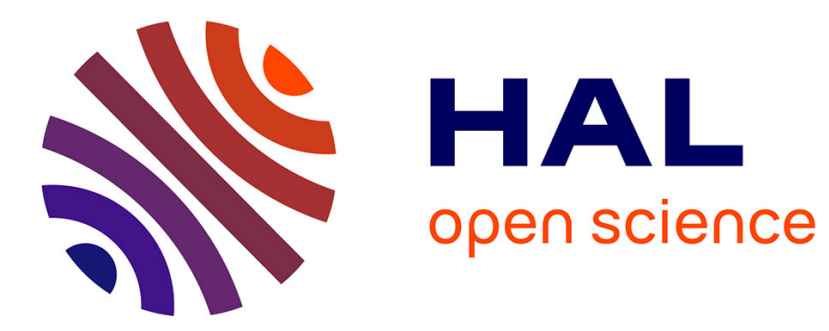

\title{
The domination number of Cartesian product of two directed paths \\ Michel Mollard
}

\section{To cite this version:}

Michel Mollard. The domination number of Cartesian product of two directed paths. Journal of Combinatorial Optimization, 2014, 27 (1), pp.144-151. hal-00655403

\section{HAL Id: hal-00655403 \\ https://hal.science/hal-00655403}

Submitted on 29 Dec 2011

HAL is a multi-disciplinary open access archive for the deposit and dissemination of scientific research documents, whether they are published or not. The documents may come from teaching and research institutions in France or abroad, or from public or private research centers.
L'archive ouverte pluridisciplinaire HAL, est destinée au dépôt et à la diffusion de documents scientifiques de niveau recherche, publiés ou non, émanant des établissements d'enseignement et de recherche français ou étrangers, des laboratoires publics ou privés. 


\title{
The domination number of Cartesian product of two directed paths
}

\author{
Michel Mollard* \\ Institut Fourier \\ 100, rue des Maths \\ 38402 St martin d'hères Cedex FRANCE \\ michel.mollard@ujf-grenoble.fr
}

\section{Introduction and definitions}

Let $\gamma\left(P_{m} \square P_{n}\right)$ be the domination number of the Cartesian product of directed paths $P_{m}$ and $P_{n}$ for $m, n \geq 2$. In [13] Liu and al. determined the value of $\gamma\left(P_{m} \square P_{n}\right)$ for arbitrary $n$ and $m \leq 6$. In this work we give the exact value of $\gamma\left(P_{m} \square P_{n}\right)$ for any $m, n$ and exhibit minimum dominating sets.

AMS Classification[2010]:05C69,05C38.

Keywords: Directed graph, digraph, Cartesian product, Domination number, Paths.

Let $G=(V, E)$ be a finite directed graph (digraph for short) without loops or multiple arcs.

A vertex $u$ dominates a vertex $v$ if $u=v$ or $u v \in E$. A set $S \subset V$ is a dominating set of $G$ if any vertex of $G$ is dominated by at least a vertex of $S$. The domination number of $G$, denoted by $\gamma(G)$ is the minimum cardinality of a dominating set. The set $V$ is a dominating set thus $\gamma(G)$ is finite. These definitions extend to digraphs the classical domination notion for undirected graphs.

The determination of domination number of a directed or undirected graph is, in general, a difficult question in graph theory. Furthermore this problem has connections with information theory. For example the domination number of Hypercubes is linked to error-correcting codes. Among the lot of related works ([7], [8]) mention the special case of domination of Cartesian product of undirected paths or cycles ([1] to [6], [9], [10]).

${ }^{*}$ CNRS Université Joseph Fourier 
For two digraphs, $G_{1}=\left(V_{1}, E_{1}\right)$ and $G_{2}=\left(V_{2}, E_{2}\right)$, the Cartesian product $G_{1} \square G_{2}$ is the digraph with vertex set $V_{1} \times V_{2}$ and $\left(x_{1}, x_{2}\right)\left(y_{1}, y_{2}\right) \in E\left(G_{1} \square G_{2}\right)$ if and only if $x_{1} y_{1} \in E_{1}$ and $x_{2}=y_{2}$ or $x_{2} y_{2} \in E_{2}$ and $x_{1}=y_{1}$. Note that $G \square H$ is isomorphic to $H \square G$.

The domination number of Cartesian product of two directed cycles have been recently investigated ([11], [12], [14], [15]). Even more recently, Liu and al.([13]) began the study of the domination number of the Cartesian product of two directed paths $P_{m}$ and $P_{n}$. They proved the following result

Theorem 1 Let $n \geq 2$. Then

- $\gamma\left(P_{2} \square P_{n}\right)=n$

- $\gamma\left(P_{3} \square P_{n}\right)=n+\left\lceil\frac{n}{4}\right\rceil$

- $\gamma\left(P_{4} \square P_{n}\right)=n+\left\lceil\frac{2 n}{3}\right\rceil$

- $\gamma\left(P_{5} \square P_{n}\right)=2 n+1$

- $\gamma\left(P_{6} \square P_{n}\right)=2 n+\left\lceil\frac{n+2}{3}\right\rceil$.

In this paper we are able to give a complete solution of the problem. In Theorem 2 we determine the value of $\gamma\left(P_{m} \square P_{n}\right)$ for any $m, n \geq 2$. When $m$ grows, the cases approach appearing in the proof of Theorem 1 seems to be more and more complicated. We proceed by a different and elementary method, but will assume that Theorem 1 is already obtained (at least for $m \leq 5$ and arbitrary $n$ ). In the next section we describe three dominating sets of $P_{m} \square P_{n}$ corresponding to the different values of $m$ modulo 3 . In the last section we prove that these dominating sets are minimum and deduce our main result:

Theorem 2 Let $n \geq 2$. Then

$$
\begin{aligned}
& \text { - } \gamma\left(P_{3 k} \square P_{n}\right)=k(n+1)+\left\lfloor\frac{n-2}{3}\right\rfloor \text { for } k \geq 2 \text { and } n \neq 3 \\
& \text { - } \gamma\left(P_{3 k+1} \square P_{n}\right)=k(n+1)+\left\lceil\frac{2 n-3}{3}\right\rceil \text { for } k \geq 1 \text { and } n \neq 3 \\
& \text { - } \gamma\left(P_{3 k+2} \square P_{n}\right)=k(n+1)+n \text { for } k \geq 0 \text { and } n \neq 3 \\
& \text { - } \gamma\left(P_{3} \square P_{n}\right)=\gamma\left(P_{n} \square P_{3}\right)=n+\left\lceil\frac{n}{4}\right\rceil .
\end{aligned}
$$

We will follow the notations used by Liu and al. and refer to their paper for a more complete description of the motivations. Let us recall some of these notations.

We denote the vertices of a directed path $P_{n}$ by the integers $\{0,1, \ldots, n-1\}$. For any $i$ in $\{0,1, \ldots, n-1\}, P_{m}^{i}$ is the subgraph of $P_{m} \square P_{n}$ induced by the vertices $\{(k, i) / k \in\{0,1, \ldots, m-1\}\}$. Note that $P_{m}^{i}$ is isomorphic to $P_{m}$. Notice also that $P_{m} \square P_{n}$ is isomorphic to $P_{n} \square P_{m}$ thus $\gamma\left(P_{m} \square P_{n}\right)=\gamma\left(P_{n} \square P_{m}\right)$. A vertex $(a, b) \in P_{m}^{b}$ can be dominated by $(a, b),(a-1, b) \in P_{m}^{b}$ (if $\left.a \geq 1\right),(a, b-1) \in P_{m}^{b-1}$ (if $b \geq 1$ ).

\section{Three Dominating sets}

We will first study $P_{3 k} \square P_{n}$ for $k \geq 1$ and $n \geq 2$. Consider the following sets of vertices of $P_{3 k}$. 
Let $D_{n}$ (see Figure 1) be the set of vertices of $P_{3 k} \square P_{n}$ consisting of the vertices

- $X=\{0,1,3,4, \ldots, 3 k-3,3 k-2\}=\{3 i / i \in\{0,1, \ldots k-1\}\} \cup\{3 i+1 / i \in$ $\{0,1, \ldots k-1\}\}$

- $Y=\{2,5,8, \ldots, 3 k-1\}=\{3 i+2 / i \in\{0,1, \ldots k-1\}\}$

- $I=\{0,3,6, \ldots, 3 k-3\}=\{3 i / i \in\{0,1, \ldots k-1\}\}$

- $J=\{1,4,7, \ldots, 3 k-2\}=\{3 i+1 / i \in\{0,1, \ldots k-1\}\}$

- $K=\{0,2,5,8 \ldots, 3 k-1\}=\{0\} \cup\{3 i+2 / i \in\{0,1, \ldots k-1\}\}$.

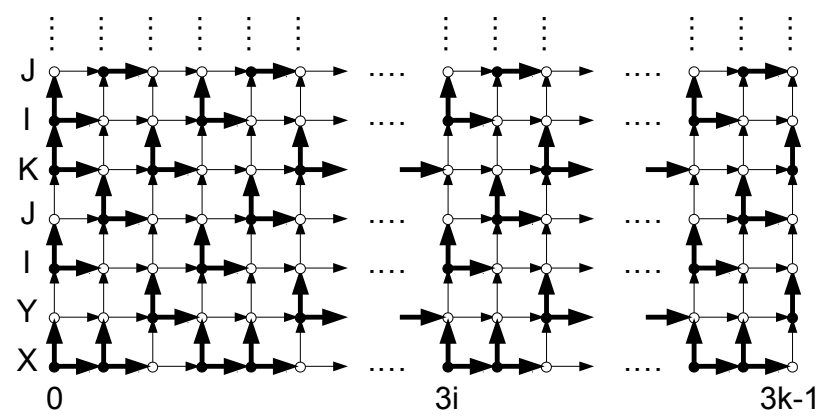

Figure 1: The dominating set $D_{n}$

Figure 1: The dominating set $D_{n}$

- $(a, 0)$ for $a \in X$

- $(a, 1)$ for $a \in Y$

- $(a, b)$ for $b \equiv 2 \bmod 3(2 \leq b<n)$ and $a \in I$

- $(a, b)$ for $b \equiv 0 \bmod 3(3 \leq b<n)$ and $a \in J$

- $(a, b)$ for $b \equiv 1 \bmod 3(4 \leq b<n)$ and $a \in K$.

Lemma 3 For any $k \geq 1, n \geq 2$ the set $D_{n}$ is a dominating set of $P_{3 k} \square P_{n}$ and $\left|D_{n}\right|=k(n+1)+\left\lfloor\frac{n-2}{3}\right\rfloor$.

Proof : It is immediate to verify that

- All vertices of $P_{3 k}$ are dominated by the vertices of $X$

- The vertices of $P_{3 k}$ not dominated by some of $Y$ are $\{0,1,4, \ldots, 3 k-2\} \subset X$

- The vertices of $P_{3 k}$ not dominated by some of $I$ are $\{2,5, \ldots, 3 k-1\}=Y \subset$ K

- The vertices of $P_{3 k}$ not dominated by some of $J$ are $\{0,3,6, \ldots, 3 k-3\} \subset I$ 
- The vertices of $P_{3 k}$ not dominated by some of $K$ are $\{4,7, \ldots, 3 k-2\} \subset J$.

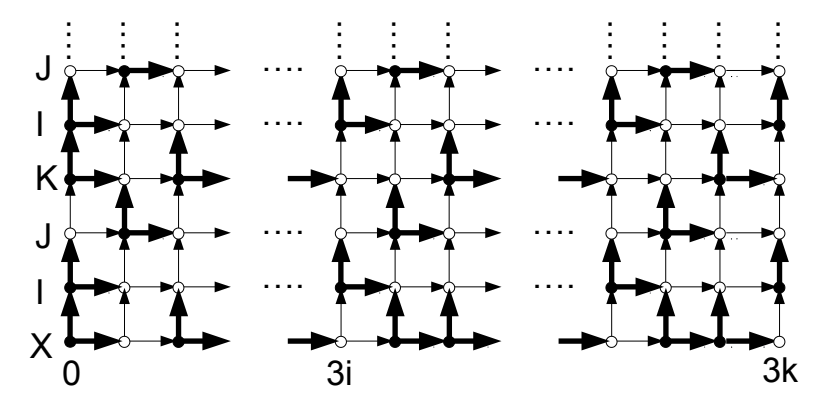

Figure 2: The dominating set $E_{n}$ thus $\left|D_{n}\right|=k(n+1)+\left\lfloor\frac{n-2}{3}\right\rfloor$. vertices of $P_{3 k+1}$. $\{3 i+1 / i \in\{1, \ldots k-1\}\}$

- $I=\{0,3,6, \ldots, 3 k\}=\{3 i / i \in\{0,1, \ldots k\}\}$

- $J=\{1,4,7, \ldots, 3 k-2\}=\{3 i+1 / i \in\{0,1, \ldots k-1\}\}$

- $K=\{0,2,5,8, \ldots, 3 k-1\}=\{0\} \cup\{3 i+2 / i \in\{0,1, \ldots k-1\}\}$.

Therefore any vertex of some $P_{3 k}^{i}$ is dominated by a vertex in $P_{3 k}^{i} \cap D_{n}$ or in $P_{3 k}^{i-1} \cap D_{n}$ (if $i \geq 1$ ). Furthermore $|X|=2 k,|Y|=|I|=|J|=k$, and $|K|=k+1$

Let us study now $P_{3 k+1} \square P_{n}$ for $k \geq 1$ and $n \geq 2$. Consider the following sets of

- $X=\{0,2,4,5,7,8, \ldots, 3 k-2,3 k-1\}=\{0\} \cup\{3 i+2 / i \in\{0,1, \ldots k-1\}\} \cup$

Let $E_{n}$ (see Figure 2) be the set of vertices of $P_{3 k+1} \square P_{n}$ consisting of the vertices

- $(a, 0)$ for $a \in X$

- $(a, b)$ for $b \equiv 1 \bmod 3(1 \leq b<n)$ and $a \in I$

- $(a, b)$ for $b \equiv 2 \bmod 3(2 \leq b<n)$ and $a \in J$

- $(a, b)$ for $b \equiv 0 \bmod 3(3 \leq b<n)$ and $a \in K$.

Lemma 4 For any $k \geq 1, n \geq 2$ the set $E_{n}$ is a dominating set of $P_{3 k+1} \square P_{n}$ and $\left|E_{n}\right|=k(n+1)+\left\lceil\frac{2 n-3}{3}\right\rceil$.

Proof : It is immediate to verify that

- All vertices of $P_{3 k+1}$ are dominated by the vertices of $X$ 
- The vertices of $P_{3 k+1}$ not dominated by some of $I$ are $\{2,5, \ldots, 3 k-1\} \subset K$ $\subset X$

- The vertices of $P_{3 k+1}$ not dominated by some of $J$ are $\{0,3,6, \ldots, 3 k\}=I$

- The vertices of $P_{3 k+1}$ not dominated by some of $K$ are $\{4,7, \ldots, 3 k-2\} \subset J$.

Therefore any vertex of some $P_{3 k+1}^{i}$ is dominated by a vertex in $P_{3 k+1}^{i} \cap E_{n}$ or

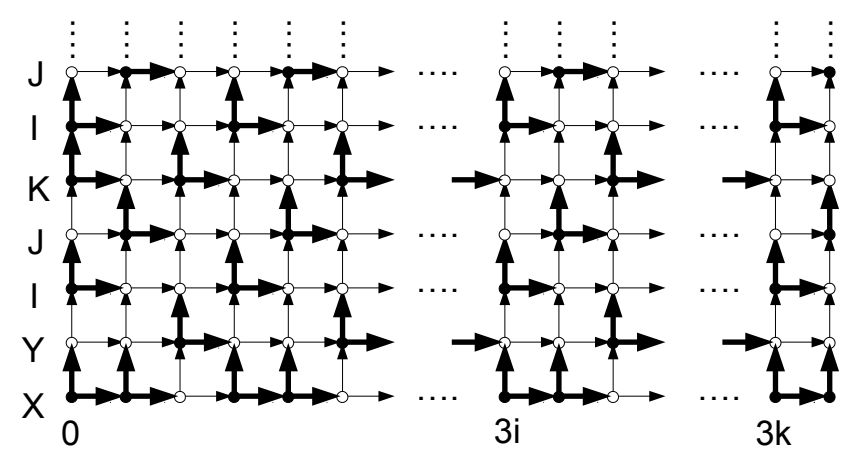

Figure 3: The dominating set $F_{n}$

Let $F_{n}$ (see Figure 3 ) be the set of vertices of $P_{3 k+2} \square P_{n}$ consisting of the vertices in $P P_{3 k+1}^{i-1} \cap E_{n}$ (if $i \geq 1$ ). Furthermore $|X|=2 k,|I|=|K|=k+1$, and $|J|=k$ thus $\left|E_{n}\right|=k(n+1)+\left\lceil\frac{2 n-3}{3}\right\rceil$.

The last case will be $P_{3 k+2} \square P_{n}$ for $k \geq 0$ and $n \geq 2$. Consider the following sets of vertices of $P_{3 k+2}$.

- $X=\{0,1,3,4, \ldots, 3 k, 3 k+1\}=\{3 i / i \in\{0,1, \ldots k\}\} \cup\{3 i+1 / i \in\{0,1, \ldots k\}\}$

- $Y=\{2,5,8, \ldots, 3 k-1\}=\{3 i+2 / i \in\{0,1, \ldots k-1\}\}$

- $I=\{0,3,6, \ldots, 3 k\}=\{3 i / i \in\{0,1, \ldots k\}\}$

- $J=\{1,4,7, \ldots, 3 k+1\}=\{3 i+1 / i \in\{0,1, \ldots k\}\}$

- $K=\{0,2,5,8 \ldots, 3 k-1\}=\{0\} \cup\{3 i+2 / i \in\{0,1, \ldots k-1\}\}$.

- $(a, 0)$ for $a \in X$

- $(a, 1)$ for $a \in Y$

- $(a, b)$ for $b \equiv 2 \bmod 3(2 \leq b<n)$ and $a \in I$

- $(a, b)$ for $b \equiv 0 \bmod 3(3 \leq b<n)$ and $a \in J$

- $(a, b)$ for $b \equiv 1 \bmod 3(4 \leq b<n)$ and $a \in K$. 
Lemma 5 For any $k \geq 0, n \geq 2$, the set $F_{n}$ is a dominating set of $P_{3 k+2} \square P_{n}$ and $\left|F_{n}\right|=k(n+1)+n$.

Proof : It is immediate to verify that

- All vertices of $P_{3 k+2}$ are dominated by the vertices of $X$

- The vertices of $P_{3 k+2}$ not dominated by some of $Y$ are $\{0,1,4, \ldots, 3 k+1\} \subset$ $X$

- The vertices of $P_{3 k+2}$ not dominated by some of $I$ are $\{2,5, \ldots, 3 k-1\}=Y \subset$ $K$

- The vertices of $P_{3 k+2}$ not dominated by some of $J$ are $\{0,3, \ldots, 3 k\}=I$

- The vertices of $P_{3 k+2}$ not dominated by some of $K$ are $\{4,7, \ldots, 3 k+1\} \subset J$.

Therefore any vertex of some $P_{3 k+2}^{i}$ is dominated by a vertex in $P_{3 k+2}^{i} \cap F_{n}$ or in $P_{3 k+2}^{i-1} \cap F_{n}$ (if $i \geq 1$ ). Furthermore $|X|=2 k+2,|Y|=k$ and $|I|=|J|=|K|=k+1$, thus $\left|F_{n}\right|=k(n+1)+n$.

\section{Optimality of the three sets}

The structure of $P_{m} \square P_{n}$ implies the following strong property.

Proposition 6 Let $S$ be a dominating set of $P_{m} \square P_{n}$. For any $n^{\prime} \leq n$ consider

$$
S_{n^{\prime}}=\bigcup_{i=0, . ., n^{\prime}-1} P_{m}^{i} \cap S
$$

Then $S_{n^{\prime}}$ is a dominating set of $P_{m} \square P_{n^{\prime}}$.

Notice that the three sets $D_{n}, E_{n}, F_{n}$ satisfy, for example, $\left(D_{n}\right)_{n^{\prime}}=D_{n^{\prime}}$ therefore we can use the same notation without ambiguity.

If $S$ is a dominating set of $P_{m} \square P_{n}$, for any $i$ in $\{0,1, \ldots, \mathrm{n}-1\}$ let $s_{i}=\left|P_{m}^{i} \cap S\right|$. We have thus $|S|=\sum_{i=0}^{n-1} s_{i}$.

Proposition 7 Let $S$ be a dominating set of $P_{m} \square P_{n}$. Let $i \in\{1,2, \ldots, n-1\}$ then $s_{i-1}+2 s_{i} \geq m$.

Proof : Any vertex of $P_{m}^{i}$ must be dominated by some vertex of $P_{m}^{i} \cap S$ or of $P_{m}^{i-1} \cap S$. A vertex in $P_{m}^{i} \cap S$ dominates at most two vertices of $P_{m}^{i}$ and a vertex in $P_{m}^{i-1} \cap S$ dominates a unique vertex of $P_{m}^{i}$.

Lemma 8 Let $k \geq 0$ and $n \geq 2, n \neq 3$, then $\gamma\left(P_{3 k+2} \square P_{n}\right)=k(n+1)+n$.

Proof : The case $n=2$ is immediate by Theorem 1 .

Let $S$ be a dominating set of $P_{3 k+2} \square P_{n}$ with $n \geq 4$.

By Proposition $7, s_{i} \leq k$ implies $s_{i-1}+s_{i} \geq m-s_{i} \geq 2 k+2$. Therefore for any $i \in\{2, \ldots, n-1\}$ we get $s_{i} \geq k+1$ or $s_{i-1}+s_{i} \geq 2(k+1)$.

Apply the following algorithm: 


$$
I:=\emptyset ; J:=\emptyset ; i:=n-1 \text {; }
$$

while $i \geq 5$ do

if $s_{i} \geq k+1$ then

$$
I:=I \cup\{i\} ; i:=i-1
$$

else

$$
J:=J \cup\{i, i-1\} ; i:=i-2
$$

end if

\section{end while}

If $n=4$ or $n=5$ the algorithm only sets $I$ and $J$ to $\emptyset$. In the general case, the algorithm stop when $i=3$ or $i=4$ and we get two disjoint sets $I, J$ with $\{0,1, \ldots, n-1\}=\{0,1,2,3\} \cup I \cup J$ or $\{0,1, \ldots, n-1\}=\{0,1,2,3,4\} \cup I \cup J$. Furthermore $\sum_{i \in I} s_{i} \geq|I|(k+1)$ and $\sum_{i \in J} s_{i} \geq|J|(k+1)$. We have thus one of the two inequalities

$$
|S|-\left(s_{0}+s_{1}+s_{2}+s_{3}\right) \geq(n-4)(k+1)
$$

or

$$
|S|-\left(s_{0}+s_{1}+s_{2}+s_{3}+s_{4}\right) \geq(n-5)(k+1) .
$$

In the first case by Proposition 6 and Theorem 1 we get $s_{0}+s_{1}+s_{2}+s_{3} \geq$ $\gamma\left(P_{3 k+2} \square P_{4}\right)=\gamma\left(P_{4} \square P_{3 k+2}\right)=3 k+2+\left\lceil\frac{6 k+4}{3}\right\rceil=5 k+4$. Thus $|S| \geq(n+1) k+n$. In the second case we get $s_{0}+s_{1}+s_{2}+s_{3}+s_{4} \geq \gamma\left(P_{5} \square P_{3 k+2}\right)=6 k+5$. Thus again $|S| \geq(n+1) k+n$.

Therefore for any $n \geq 4$ we have $\gamma\left(P_{3 k+2} \square P_{n}\right) \geq k(n+1)+n$ and the equality occurs by Lemma 5 .

Notice that, by Theorem 1, $\gamma\left(P_{3 k+2} \square P_{3}\right)=3 k+2+\left\lceil\frac{3 k+2}{4}\right\rceil \neq 4 k+3$ for $k \geq 1$.

Lemma 9 Let $k \geq 1$ and $n \geq 2, n \neq 3$, then $\gamma\left(P_{3 k+1} \square P_{n}\right)=k(n+1)+\left\lceil\frac{2 n-3}{3}\right\rceil$.

Proof : Consider some fixed $k \geq 1$. Notice first that by Theorem $1, \gamma\left(P_{3 k+1} \square P_{2}\right)=$ $3 k+1, \gamma\left(P_{3 k+1} \square P_{4}\right)=5 k+2$ and $\gamma\left(P_{3 k+1} \square P_{5}\right)=6 k+3$ thus the result is true for $n \leq 5$.

We knows, by Lemma 4 , that for any $n \geq 2$ the set $E_{n}$ is a dominating set of $P_{3 k+1} \square P_{n}$ and $\left|E_{n}\right|=(n+1) k+\left\lceil\frac{2 n-3}{3}\right\rceil$.

We will prove now that $E_{n}$ is a minimum dominating set .

If this is not true consider $n$ minimum, $n \geq 2$, such that there exists a dominating set $S$ of $P_{3 k+1} \square P_{n}$ with $|S|<\left|E_{n}\right|$. We knows that $n \geq 6$.

For $n^{\prime} \leq n$ let $S_{n^{\prime}}=\cup_{i=0, . ., n^{\prime}-1} P_{3 k+1}^{i} \cap S$ and $s_{n^{\prime}}=\left|P_{3 k+1}^{n^{\prime}} \cap S\right|$.

Case $1 n=3 p, p \geq 2$.

Notice first that $\left|E_{n}\right|-\left|E_{n-1}\right|=k$ and $\left|E_{n}\right|-\left|E_{n-2}\right|=2 k+1$. We have also by hypothesis $|S| \leq\left|E_{n}\right|-1$. By minimality of $n, E_{n-1}$ is minimum thus $\left|S_{n-1}\right| \geq$ $\left|E_{n-1}\right|$. Therefore $s_{n-1}=|S|-\left|S_{n-1}\right| \leq\left|E_{n}\right|-1-\left|E_{n-1}\right|=k-1$. On the other hand, by Proposition $7, s_{n-2}+2 s_{n-1} \geq 3 k+1$ thus $s_{n-2}+s_{n-1} \geq(3 k+1)-(k-1)=2 k+2$. This implies $\left|S_{n-2}\right| \leq\left|S_{n}\right|-2 k-2 \leq\left|E_{n}\right|-2 k-3<\left|E_{n}\right|-2 k-1=\left|E_{n-2}\right|$, thus $E_{n-2}$ is not minimum in contradiction with $n$ minimum. 
Case $2 n=3 p+1, p \geq 2$.

In this case we have $\left|E_{n}\right|-\left|E_{n-1}\right|=k+1$ and $\left|E_{n}\right|-\left|E_{n-2}\right|=2 k+1$. We have also by hypothesis $|S| \leq\left|E_{n}\right|-1$. By minimality of $n, E_{n-1}$ is minimum thus $\left|S_{n-1}\right| \geq\left|E_{n-1}\right|$. Therefore $s_{n-1}=|S|-\left|S_{n-1}\right| \leq\left|E_{n}\right|-1-\left|E_{n-1}\right|=k$. On the other hand, by Proposition $7, s_{n-2}+2 s_{n-1} \geq 3 k+1$ thus $s_{n-2}+s_{n-1} \geq 2 k+1$. This implies $\left|S_{n-2}\right| \leq\left|S_{n}\right|-2 k-1<\left|E_{n}\right|-2 k-1=\left|E_{n-2}\right|$, thus $E_{n-2}$ is not minimum in contradiction with $n$ minimum.

Case $3 n=3 p+2, p \geq 2$.

In this case, $\left|E_{n}\right|-\left|E_{n-2}\right|=2 k+2$ and we cannot proceed like case 1 and case 2 . Hopefully, by Lemma 8, $\gamma\left(P_{3 k+1} \square P_{3 p+2}\right)=\gamma\left(P_{3 p+2} \square P_{3 k+1}\right)=p(3 k+2)+3 k+1=$ $k(3 p+3)+2 p+1$. Therefore, since $n+1=3 p+3$ and $\left\lceil\frac{2 n-3}{3}\right\rceil=2 p+1, E_{n}$ is minimum.

Lemma 10 Let $k \geq 2$ and $n \geq 2, n \neq 3$ then $\gamma\left(P_{3 k} \square P_{n}\right)=k(n+1)+\left\lfloor\frac{n-2}{3}\right\rfloor$.

\section{Proof :}

Case $1 n=3 p+1, p \geq 1$.

By Lemma $9, \gamma\left(P_{3 k} \square P_{3 p+1}\right)=\gamma\left(P_{3 p+1} \square P_{3 k}\right)=p(3 k+1)+2 k-1=k(3 p+2)+p-1$. We obtain the conclusion since $3 p+2=n+1$ and $\left\lfloor\frac{n-2}{3}\right\rfloor=p-1$.

Case $2 n=3 p+2, p \geq 0$.

By Lemma 8, $\gamma\left(P_{3 k} \square P_{3 p+2}\right)=\gamma\left(P_{3 p+2} \square P_{3 k}\right)=p(3 k+1)+3 k=k(3 p+3)+p$. We obtain again the conclusion since $3 p+3=n+1$ and $\left\lfloor\frac{n-2}{3}\right\rfloor=p$.

Case $3 n=3 p, p \geq 2$.

We knows, by Lemma 3 , that the set $D_{n}$ is a dominating set of $P_{3 k} \square P_{n}$ and $\left|D_{n}\right|=$ $k(n+1)+\left\lfloor\frac{n-2}{3}\right\rfloor$.

If $D_{n}$ is not a minimum dominating set let $S$ be a dominating set with $|S|<\left|D_{n}\right|$. For $n^{\prime} \leq n$ let $S_{n^{\prime}}=\cup_{i=0, . ., n^{\prime}-1} P_{3 k}^{i} \cap S$ and $s_{n^{\prime}}=\left|P_{3 k}^{n^{\prime}} \cap S\right|$.

Because $n=3 p$ and $p \geq 2$ we get $\left|D_{n}\right|-\left|D_{n-1}\right|=k$ and $\left|D_{n}\right|-\left|D_{n-2}\right|=2 k+1$. We have also by hypothesis $|S| \leq\left|D_{n}\right|-1$. Notice that, by Lemma $8, \gamma\left(P_{3 k} \square P_{n-1}\right)=$ $\gamma\left(P_{3 p-1} \square P_{3 k}\right)=(p-1)(3 k+1)+3 k=k n+\left\lfloor\frac{n-3}{3}\right\rfloor=\left|D_{n-1}\right|$ thus $D_{n-1}$ is minimum and $\left|S_{n-1}\right| \geq\left|D_{n-1}\right|$.

Therefore $s_{n-1}=|S|-\left|S_{n-1}\right| \leq\left|D_{n}\right|-1-\left|D_{n-1}\right|=k-1$. By Proposition $7, s_{n-2}+2 s_{n-1} \geq 3 k$ thus $s_{n-2}+s_{n-1} \geq 2 k+1$. This implies $\left|S_{n-2}\right| \leq$ $|S|-2 k-1<\left|D_{n}\right|-2 k-1=\left|D_{n-2}\right|$. On the other hand, by Lemma 9, $\gamma\left(P_{3 k} \square P_{3 p-2}\right)=\gamma\left(P_{3 p-2} \square P_{3 k}\right)=(p-1)(3 k+1)+2 k-1=k(n-1)+\left\lfloor\frac{n-4}{3}\right\rfloor=\left|D_{n-2}\right|$ thus $D_{n-2}$ is minimum, a contradiction.

Notice that, by Theorem $1, \gamma\left(P_{3 k} \square P_{3}\right)=3 k+\left\lceil\frac{3 k}{4}\right\rceil \neq 4 k$ for $k \geq 3$.

\section{Conclusions}

Putting together Lemma 8, Lemma 9, Lemma 10 and the case $m=3$ or $n=3$, we obtain $\gamma\left(P_{m} \square P_{n}\right)$ for any $m, n$ (Theorem 2 ).

As a conclusion, notice that the minimum dominating sets we build for $P_{5} \square P_{n}$ and 
$P_{6} \square P_{n}$ are different than those proposed by Liu and al.([13]). An open problem would be to characterize all minimum dominating sets of $P_{m} \square P_{n}$.

\section{References}

[1] T.Y. Chang, W.E. Clark: "The Domination numbers of the $5 \times n$ and $6 \times n$ grid graph", J.Graph Theory, 17 (1993) 81-107.

[2] M. El-Zahar, C.M. Pareek: "Domination number of products of graphs", Ars Combin., 31 (1991) 223-227.

[3] M. El-Zahar, S. Khamis, Kh. Nazzal: "On the Domination number of the Cartesian product of the cycle of length $n$ and any graph", Discrete App. Math., 155 (2007) 515-522.

[4] R.J. Faudree, R.H. Schelp: 'The Domination number for the product of graphs", Congr. Numer., 79 (1990) 29-33.

[5] S. Gravier, M. Mollard: "On Domination numbers of Cartesian product of paths", Discrete App. Math., 80 (1997) 247-250.

[6] B. Hartnell, D. Rall: "On dominating the Cartesian product of a graph and $K_{2} "$, Discuss. Math. Graph Theory, 24(3) (2004) 389-402.

[7] T.W. Haynes, S.T. Hedetniemi, P.J. Slater: Fundamentals of Domination in Graphs, Marcel Dekker, Inc. New York, 1998.

[8] T.W. Haynes, S.T. Hedetniemi, P.J. Slater eds.: Domination in Graphs: Advanced Topics, Marcel Dekker, Inc. New York, 1998.

[9] M.S. Jacobson, L.F. Kinch: "On the Domination number of products of graphs I", Ars Combin., 18 (1983) 33-44.

[10] S. Klavžar, N. Seifter: "Dominating Cartesian products of cycles", Discrete App. Math., 59 (1995) 129-136.

[11] J. Liu, X.D. Zhang, X. Chen, J.Meng: "The Domination number of Cartesian products of directed cycles", Inf. Process. Lett., 110(5) (2010) 171-173.

[12] J. Liu, X.D. Zhang, X. Chen, J.Meng: "On Domination number of Cartesian product of directed cycles", Inf. Process. Lett., 111(1) (2010) 36-39.

[13] J. Liu, X.D. Zhang, X. Chen, J.Meng: "On Domination number of Cartesian product of directed paths", J. Comb. Optim., 22(4) (2011) 651-662.

[14] R.S. Shaheen: "Domination number of toroidal grid digraphs", Utilitas Mathematica 78(2009) 175-184.

[15] M.Mollard: "On Domination of Cartesian product of directed cycles", submitted (2011). Manuscript available on line: http://hal.archives-ouvertes.fr/hal$00576481 / \mathrm{fr} /$. 